Gut and Liver, Vol. 10, No. 1, January 2016, pp. 37-41

\title{
Clinicopathologic Analysis of Proton Pump Inhibitor-Responsive Esophageal Eosinophilia in Korean Patients
}

\author{
Da Hyun Jung*, Gak-Won Yun*, Yoo Jin Lee*, Yunju Jo ${ }^{\dagger}$, and Hyojin Park* \\ ${ }^{*}$ Department of Internal Medicine, Gangnam Severance Hospital, Yonsei University College of Medicine, and ${ }^{\dagger}$ Department of Internal Medicine, \\ Eulji University School of Medicine, Seoul, Korea
}

See editorial on page 1.

Background/Aims: Proton pump inhibitor-responsive esophageal eosinophilia (PPI-REE) is a newly recognized form of eosinophilic esophagitis (EoE) that responds to PPI therapy. It remains unclear whether PPI-REE represents a subphenotype of gastroesophageal reflux disease, a subphenotype of EoE, or its own distinct entity. The aim was to evaluate the clinicopathologic features of PPI-REE. Methods: Six patients were diagnosed with PPI-REE based on symptoms, endoscopic abnormalities, esophageal eosinophilia with $\geq 15$ eosinophils/high-power field, and a response to PPI treatment. Symptoms and endoscopic and pathological findings were evaluated. Results: The median follow-up duration was 12 months. Presenting symptoms included dysphagia, heartburn, chest pain, foreign body sensation, acid reflux, and sore throat. All patients had typical endoscopic findings of EoE such as esophageal rings, linear furrows, nodularity, and whitish plaques. Three patients had a concomitant allergic disorder, and one had reflux esophagitis. Four patients exhibited elevated serum lgE, and five had positive skin prick tests. All patients experienced symptomatic resolution within 4 weeks and histologic resolution within 8 weeks after starting PPI therapy. There was no symptomatic recurrence. Conclusions: PPI therapy induced rapid resolution of symptoms and eosinophil counts in patients with PPI-REE. Largescale studies with long-term follow-up are warranted. (Gut Liver 2016;10:37-41)

Key Words: Proton pump inhibitor responsive esophageal eosinophilia; Eosinophilic esophagitis; Proton pump inhibitors

\section{INTRODUCTION}

Eosinophilic esophagitis (EoE) is a chronic inflammatory disorder characterized by eosinophilic infiltration in the esophagus, in which its occurrence has been increasingly recognized in Western countries. ${ }^{1,2}$ However, esophageal eosinophilia is not specific for EoE. Esophageal eosinophilic infiltration (EEI) occurs in a number of conditions, including infections, drug hypersensitivity, gastroesophageal reflux disease (GERD), celiac disease, Crohn's disease, hypereosinophilic syndrome, connective tissue diseases, and graft-versus-host disease. ${ }^{3}$ Among other conditions, GERD and proton pump inhibitor-responsive esophageal eosinophilia (PPI-REE) are common diseases that must be differentiated from EoE. ${ }^{3-5}$

PPI-REE is a newly described disease. It is diagnosed when patients have esophageal symptoms and histologic features of esophageal eosinophilia but exhibit symptomatic and histologic improvement with PPI therapy. ${ }^{3}$ The first case series of three pediatric patients whose symptoms and eosinophilia resolved with PPI treatment was reported by Ngo et al. ${ }^{6}$ in 2006.

Retrospective studies have demonstrated that 39\% to 71\% of children and adults with EEI have PPI-REE. ${ }^{7-11}$ However, there have been very few reports regarding PPI-REE in Asian populations. A recent study from Japan reported that the prevalence of EoE and PPI-REE was two (0.01\%) and three (0.02\%) of 13,634 patients, respectively, who underwent upper gastrointestinal (GI) endoscopy. ${ }^{12}$ Another Japanese case series of 12 patients with EEI reported that after PPI treatment, two patients (28.6\%) were diagnosed with EoE and five patients (71.4\%) were diagnosed with PPI-REE. ${ }^{13}$ A recent American College of Gastroenterology guideline recommends that patients suspected of EoE be given PPI treatment to exclude PPI-REE. In addition, the guideline recommends additional evaluation, such as ambulatory $\mathrm{pH}$

Correspondence to: Hyojin Park

Department of Internal Medicine, Gangnam Severance Hospital, Yonsei University College of Medicine, 211 Eonju-ro, Gangnam-gu, Seoul 06273, Korea

Tel: +82-2-2019-3318, Fax: +82-2-3463-3882, E-mail: hjpark21@yuhs.ac

Received on July 18, 2014. Revised on October 10, 2014. Accepted on November 14, 2014. Published online May 13,2015 pISSN 1976-2283 eISSN 2005-1212 http://dx.doi.org/10.5009/gnl14269

@ This is an Open Access article distributed under the terms of the Creative Commons Attribution Non-Commercial License (http://creativecommons.org/licenses/by-nc/4.0) which permits unrestricted non-commercial use, distribution, and reproduction in any medium, provided the original work is properly cited. 
monitoring, to determine whether GERD is the cause of EEI. ${ }^{3}$

Despite the increasing recognition of PPI-REE, it is unclear whether PPI-REE is a GERD variant, an EoE variant, or an independent disease entity. A recent prospective cohort study from the United States reported that clinical, endoscopic, and histologic features could not distinguish PPI-REE from EoE before a trial using PPI. ${ }^{14}$ The aim of this study was to investigate the clinical, endoscopic, and histologic characteristics of PPI-REE in Korean adult patients.

\section{MATERIALS AND METHODS}

\section{Patients}

We reviewed the records of 20 patients diagnosed with EEI at four tertiary university hospitals in urban areas between September 2005 and May 2013. The diagnosis cutoff value for EEI was defined as a peak of $\geq 15$ eosinophils per high-power field (HPF) on esophageal biopsy. Patients were diagnosed with EoE if they met these criteria: (1) presence of symptoms related to esophageal dysfunction; (2) mucosal eosinophilia in a esophageal biopsy with at least 15 eosinophils per HPF, which persisted even after a PPI therapeutic regimen; and (3) absence of other causes of esophageal eosinophilia. Patients were diagnosed with PPI-REE if they had esophageal symptoms and histologic features of esophageal eosinophilia, and resolution of symptoms and histologic features after a 2-month course of a PPI therapeutic regimen. ${ }^{3}$ Baseline patient characteristics were recorded, including age, gender, and allergic history (e.g., food allergy, allergic dermatitis, allergic rhinitis, and asthma). The presence of these symptoms was noted: food impaction, dysphagia, heartburn, chest pain, and sore throat. The peripheral eosinophil count and serum IgE levels were recorded. Endoscopic findings of suspected EoE included mucosal rings, linear furrows, white exudates, friability, and stricture. When present, reflux esophagitis was diagnosed and graded. Two to four biopsies specimens were obtained from the mid and distal esophagus.

Treatment of EEI consisted of PPI and/or inhaled (fluticasone propionate) or oral corticosteroid for 4 to 8 weeks. During PPI treatment, patients did not follow the six-food elimination diet. This study was approved by the Institutional Review Board of Gangnam Severance Hospital, Yonsei University College of Medicine (3-2013-0249).

\section{Immunohistochemistry}

Biopsy specimens obtained from the esophagus of three patients before and after PPI treatment were used for immunohistochemical staining. We used paraffin-embedded tissue specimens and antihuman CCL26 goat polyclonal antibody (AF653; R\&D Systems, Minneapolis, MN, USA).

\section{RESULTS}

\section{Patient flow and characteristics of subjects with PPI-REE}

Of the 20 patients with EEI, 11 (55.0\%) did not undergo endoscopy at the time of follow-up. After PPI treatment, six (30.0\%) were diagnosed with PPI-REE and three (15.0\%) were diagnosed

Table 1. Baseline Characteristics of Patients with Proton Pump Inhibitor-Responsive Esophageal Eosinophilia

\begin{tabular}{|c|c|c|c|c|c|c|c|}
\hline $\begin{array}{c}\text { Case } \\
\text { no. }\end{array}$ & $\begin{array}{l}\text { Age, } \\
\text { yr }\end{array}$ & Sex & Symptom & Allergic condition & $\begin{array}{c}\text { Peripheral } \\
\text { eosinophilia, } / \mu \mathrm{L}\end{array}$ & $\begin{array}{l}\text { Serum IgE, } \\
\text { kIU/L }\end{array}$ & $\begin{array}{c}\text { Skin } \\
\text { prick test }\end{array}$ \\
\hline 1 & 54 & $\mathrm{~F}$ & Dysphagia, heartburn, foreign body sense, acid reflux & None & 130 & 16 & Negative \\
\hline 2 & 46 & M & Dysphagia & Unknown factor & 210 & $>1,000$ & Positive \\
\hline 3 & 19 & M & Dysphagia, foreign body sense, acid reflux, sore throat & Asthma, allergic rhinitis & 450 & $>1,000$ & Positive \\
\hline 4 & 24 & M & Heartburn & Food & 470 & 101.9 & Positive \\
\hline 5 & 30 & M & Heartburn, foreign body sense, acid reflux & None & 80 & 269 & Positive \\
\hline 6 & 28 & M & Dysphagia, chest pain, foreign body sense & None & 278 & 147 & Positive \\
\hline
\end{tabular}

F, female; M, male.

Table 2. Endoscopic and Histological Findings of Patients with Proton Pump Inhibitor-Responsive Esophageal Eosinophilia

\begin{tabular}{|c|c|c|c|c|c|c|}
\hline \multirow{2}{*}{ Case no. } & \multicolumn{4}{|c|}{ Endoscopic findings } & \multirow{2}{*}{ No. of eosinophils, /HPF } & \multirow{2}{*}{ Concomittent reflux esophagitis } \\
\hline & Concentric rings & Linear furrows & Nodularity & Whitish plaque & & \\
\hline 1 & + & + & - & - & 36 & None \\
\hline 2 & + & + & - & - & 194 & None \\
\hline 3 & - & + & - & + & $>20$ & None \\
\hline 4 & - & + & - & - & 21 & LA-B \\
\hline 5 & + & - & - & - & 124 & None \\
\hline 6 & - & + & + & - & $>20$ & None \\
\hline
\end{tabular}

HPF, high-power field; LA, Los Angeles classification. 
with EoE. The baseline characteristics of patients with PPI-REE are shown in Table 1. Five patients with PPI-REE were men and one was a woman. Their mean age was 33.5 years (range, 19 to 43 years). Among six PPI-REE patients, dysphagia was present in four (66.7\%), heartburn in two (33.3\%), chest pain in one (16.7\%), foreign body sensation in four (66.7\%), acid reflux in three (50.0\%), and sore throat in one (16.7\%). Three patients with PPI-REE had an allergic history and four had an elevated serum IgE. Five patients exhibited positive skin prick tests to various allergens. None had peripheral eosinophilia.

Table 2 presents the endoscopic and histologic findings of the patients with PPI-REE. All patients showed typical endoscopic findings. Esophageal rings were found in three patients (50.0\%), linear furrows in five (83.3\%), nodularity in one (16.7\%), and whitish plaques in one (16.7\%). Concomittent reflux esophagitis corresponding to Los Angeles classification grade B was observed in one (16.7\%) patient. The number of infiltrated eosinophils ranged from 21 to 194 per HPF.
The results of treatment outcomes of patients with PPIREE are presented in Table 3. Six patients with PPI-REE were received a standard dose of PPI once daily. All patients had resolution of symptoms within 4 weeks after PPI therapy and resolution of histologic changes within 8 weeks after PPI therapy. Endoscopic findings changed diversely after PPI treatment. There was no symptomatic recurrence during follow-up. The median follow-up duration was 12 months (range, 2 to 61 months).

\section{Immunohistochemistry for eotaxin-3}

Esophageal tissue of three patients with PPI-REE was stained for eotaxin-3 before and after PPI treatment. Eotaxin-3 was expressed in the esophagus of patients with PPI-REE before PPI treatment; however, it was expressed only weakly after PPI treatment (Fig. 1).

Table 3. Treatment Outcomes of Patients with Proton Pump Inhibitor-Responsive Esophageal Eosinophilia

\begin{tabular}{ccccc}
\hline Case no. & Interval of follow up endoscopy, mo & Therapeutic agents* & Esophageal eosinophilia & Symptom resolution \\
\hline 1 & 2 & Rabeprazole & Disappearance & Yes \\
2 & 3 & Esomeprazole & Decrease & Yes \\
3 & 2 & Pantoprazole & Disappearance & Yes \\
4 & 3 & Esomeprazole & Disappearance & Yes \\
5 & 2 & Esomeprazole & Decrease & Yes \\
6 & 2 & Pantoprazole & Decrease & Yes \\
\hline
\end{tabular}

*Six patients with proton pump inhibitor-responsive esophageal eosinophilia received a standard dose of proton pump inhibitor once daily.

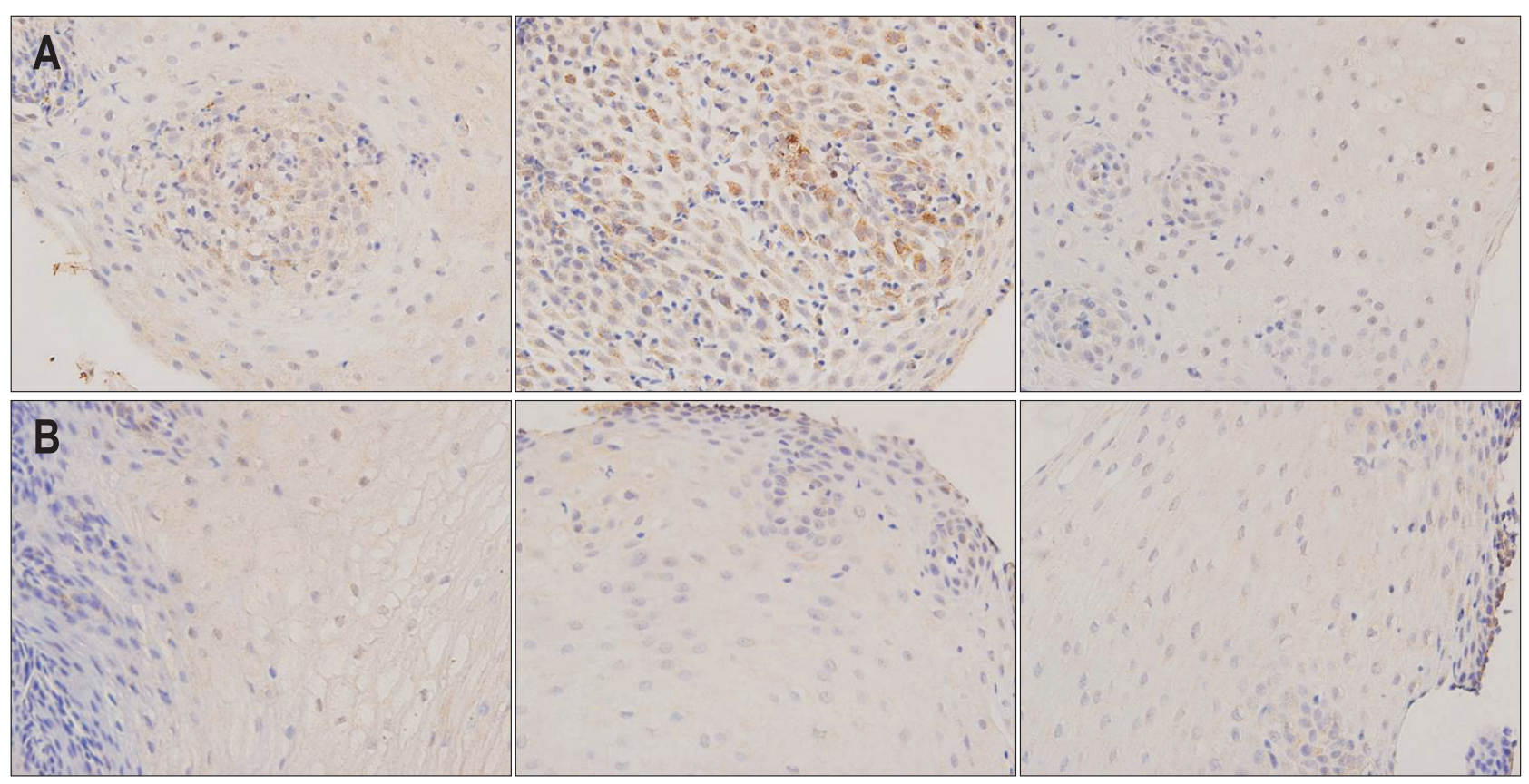

Fig. 1. Immunohistochemical staining of eotaxin-3 in proton pump inhibitor-responsive esophageal eosinophilia (PPI-REE) ( $\times 400)$. Eotaxin-3 was fully expressed in the esophagus of patients with PPI-REE before PPI treatment (A) and was expressed only weakly after PPI treatment (B). 


\section{DISCUSSION}

The concept of PPI-REE was recently recognized. However, the clinicopathologic characteristics and pathogenesis of PPIREE are not well understood. Dellon et al. ${ }^{14}$ reported that 24 of 66 patients (36.4\%) with EEI had PPI-REE after a PPI trial and that no clinical or endoscopic findings independently distinguished PPI-REE from EoE. It remains uncertain whether PPIREE is a subtype of EoE, a subtype of GERD, or an independent disease entity. It also remains unknown whether some patients with PPI-REE will eventually be categorized as belonging to a subtype of EoE over time. One case report showed that four pediatric patients with transient PPI-REE eventually developed recurrent endoscopic abnormalities and panesophageal eosinophilia despite continued PPI treatment. The authors thereby recommended that patients with PPI-REE be monitored closely for evidence of EoE because PPI-REE represented a subphenotype of EoE. ${ }^{15}$

There have been many studies of PPI-REE from Western countries. ${ }^{7-11}$ However, there have been few reports regarding PPI-REE in Asia and no previous report about the incidence or clinicopathologic characteristics of PPI-REE in Korea. A recent Japanese multicenter study demonstrated that the prevalence of EEI was seven $(0.05 \%)$ of 13,634 Japanese patients who underwent upper GI endoscopy. Among these seven patients, three (0.02\%) had PPI-REE. Another study from Japan reported that four of 23,346 patients who underwent routine GI endoscopy in 17 institutions were endoscopically and histologically diagnosed with EoE. Among these four patients, two had a partial symptomatic response after PPI administration. ${ }^{16}$ Reasons for the low prevalence of PPI-REE in Asia have not been established, although ethnic differences might be an important factor. And, there was no clinicopathologic characteristics independently distinguished PPI-REE from EoE. In this study, the clinical or endoscopic findings were similar between the patients with PPI-REE and EoE. And, the clinicopathologic characteristics of patients with PPI-REE in Korea were comparable with previous Western reports.

The symptomatic and histologic responses to PPI treatment have been attributed to several mechanisms. First, some patients with PPI-REE may have concomitant GERD. Acid suppression may therefore reduce the symptoms and eosinophilic infiltration of patients with PPI-REE. And, PPI had an effect to attenuate the permeability of the esophageal wall and thereby decrease antigen exposure. ${ }^{17,18}$ Second, it is possible that PPIs have direct eosinophil-reducing and anti-inflammatory effects independent of their effect on gastric acid secretion. ${ }^{19-21}$ Others have suggested that PPI may decrease eosinophil degranulation and improve symptoms of patients with EoE despite the persistence of eosinophilic inflammation. ${ }^{22}$

Eotaxin-3 is a potent eosinophil chemoattractant. Previous reports have indicated that patients with EoE have greater esophageal mucosal expression of eotaxin-3 mRNA and exhibit profound upregulation of the eotaxin-3 gene in esophageal mucosal biopsy specimens. ${ }^{23,24}$ Therefore, it has been suggested that PPI may improve PPI-REE by a mechanism involving esophageal expression of eotaxin-3 in EoE. Zhang et al. ${ }^{20}$ and Cheng et al. ${ }^{21}$ demonstrated that PPI blocks eotaxin-3 expression. Recent data (available only in abstract form) reported that eotaxin-3 was reduced in PPI-REE patients similar to the decrease noted in EoE patients after steroid treatment. ${ }^{25}$ Although we performed immunohistochemistry staining in only three patients, we found similar results: eotaxin-3 was expressed in the esophagus of patients with PPI-REE before PPI treatment, but only weakly expressed after PPI treatment.

Although this retrospective study was limited by the small number of patients with PPI-REE and the variable number and sites of the esophageal biopsies, this is the first study to report the clinicopathologic characteristics of patients with PPI-REE in Korea. And, the clinicopathologic characteristics of patients with PPI-REE in Korea were similar in Western. A prospective, multicenter study examining the prevalence and clinicopathologic characteristics of PPI-REE in this patient population is required.

\section{CONFLICTS OF INTEREST}

No potential conflict of interest relevant to this article was reported.

\section{REFERENCES}

1. Prasad GA, Talley NJ, Romero Y, et al. Prevalence and predictive factors of eosinophilic esophagitis in patients presenting with dysphagia: a prospective study. Am J Gastroenterol 2007;102:26272632.

2. Veerappan GR, Perry JL, Duncan TJ, et al. Prevalence of eosinophilic esophagitis in an adult population undergoing upper endoscopy: a prospective study. Clin Gastroenterol Hepatol 2009;7:420426

3. Dellon ES, Gonsalves N, Hirano I, et al. ACG clinical guideline: evidenced based approach to the diagnosis and management of esophageal eosinophilia and eosinophilic esophagitis (EoE). Am J Gastroenterol 2013;108:679-692.

4. Liacouras CA, Furuta GT, Hirano I, et al. Eosinophilic esophagitis: updated consensus recommendations for children and adults. J Allergy Clin Immunol 2011;128:3-20.

5. Rodrigo S, Abboud G, Oh D, et al. High intraepithelial eosinophil counts in esophageal squamous epithelium are not specific for eosinophilic esophagitis in adults. Am J Gastroenterol 2008;103:435442

6. Ngo P, Furuta GT, Antonioli DA, Fox VL. Eosinophils in the esophagus: peptic or allergic eosinophilic esophagitis? Case series of three patients with esophageal eosinophilia. Am J Gastroenterol 2006;101:1666-1670. 
7. Dranove JE, Horn DS, Davis MA, Kernek KM, Gupta SK. Predictors of response to proton pump inhibitor therapy among children with significant esophageal eosinophilia. J Pediatr 2009;154:96100.

8. Sayej WN, Patel R, Baker RD, Tron E, Baker SS. Treatment with high-dose proton pump inhibitors helps distinguish eosinophilic esophagitis from noneosinophilic esophagitis. J Pediatr Gastroenterol Nutr 2009;49:393-399.

9. Molina-Infante J, Ferrando-Lamana L, Ripoll C, et al. Esophageal eosinophilic infiltration responds to proton pump inhibition in most adults. Clin Gastroenterol Hepatol 2011;9:110-117.

10. Francis DL, Foxx-Orenstein A, Arora AS, et al. Results of ambulatory $\mathrm{pH}$ monitoring do not reliably predict response to therapy in patients with eosinophilic oesophagitis. Aliment Pharmacol Ther 2012;35:300-307.

11. Schroeder S, Capocelli KE, Masterson JC, et al. Effect of proton pump inhibitor on esophageal eosinophilia. J Pediatr Gastroenterol Nutr 2013;56:166-172.

12. Fujiwara Y, Sugawa T, Tanaka F, et al. A multicenter study on the prevalence of eosinophilic esophagitis and PPI-responsive esophageal eosinophilic infiltration. Intern Med 2012;51:3235-3239.

13. Abe Y, Iijima K, Ohara S, et al. A Japanese case series of 12 patients with esophageal eosinophilia. J Gastroenterol 2011;46:2530.

14. Dellon ES, Speck O, Woodward K, et al. Clinical and endoscopic characteristics do not reliably differentiate PPI-responsive esophageal eosinophilia and eosinophilic esophagitis in patients undergoing upper endoscopy: a prospective cohort study. Am J Gastroenterol 2013;108:1854-1860.

15. Dohil R, Newbury RO, Aceves S. Transient PPI responsive esophageal eosinophilia may be a clinical sub-phenotype of pediatric eosinophilic esophagitis. Dig Dis Sci 2012;57:1413-1419.

16. Fujishiro H, Amano Y, Kushiyama Y, Ishihara S, Kinoshita Y.
Eosinophilic esophagitis investigated by upper gastrointestinal endoscopy in Japanese patients. J Gastroenterol 2011;46:1142-1144.

17. De Jonge PJ, Siersema PD, Van Breda SG, et al. Proton pump inhibitor therapy in gastro-oesophageal reflux disease decreases the oesophageal immune response but does not reduce the formation of DNA adducts. Aliment Pharmacol Ther 2008;28:127-136.

18. Krarup AL, Villadsen GE, Mejlgaard E, Olesen SS, Drewes AM, Funch-Jensen P. Acid hypersensitivity in patients with eosinophilic oesophagitis. Scand J Gastroenterol 2010;45:273-281.

19. Kedika RR, Souza RF, Spechler SJ. Potential anti-inflammatory effects of proton pump inhibitors: a review and discussion of the clinical implications. Dig Dis Sci 2009;54:2312-2317.

20. Zhang X, Cheng E, Huo X, et al. Omeprazole blocks STAT6 binding to the eotaxin-3 promoter in eosinophilic esophagitis cells. PLoS One 2012;7:e50037.

21. Cheng E, Zhang X, Huo X, et al. Omeprazole blocks eotaxin-3 expression by oesophageal squamous cells from patients with eosinophilic oesophagitis and GORD. Gut 2013;62:824-832.

22. Lu Y, Levine JJ, Edelman M, Carreon CK. Symptomatic improvement in children with eosinophilic esophagitis treated with proton pump inhibitors may be due to decreased eosinophil degranulation. Gastroenterology 2013;144(5 Suppl 1):S489.

23. Bhattacharya B, Carlsten J, Sabo E, et al. Increased expression of eotaxin-3 distinguishes between eosinophilic esophagitis and gastroesophageal reflux disease. Hum Pathol 2007;38:1744-1753.

24. Blanchard C, Wang N, Stringer KF, et al. Eotaxin-3 and a uniquely conserved gene-expression profile in eosinophilic esophagitis. $\mathrm{J}$ Clin Invest 2006;116:536-547.

25. Molina-Infante J, Rivas MD, Rodriguez GV, et al. Remission in proton pump inhibitors-responsive esophageal eosinophilia correlates with downregulation of Eotaxin-3 and TH2 cytokines, similarly to eosinophilic esophagitis after steroids. Gastroenterology 2013;144(5 Suppl 1):S484. 